\title{
Selective Elimination of Cross-Compartmental Innervation in Rat Lateral Gastrocnemius Muscle
}

\author{
Sean P. Donahue' and Arthur Wm. English',2 \\ 'Department of Anatomy and Cell Biology, ${ }^{2}$ Yerkes Regional Primate Research Center, Atlanta, Georgia 30322
}

\begin{abstract}
The calf muscles of the rat hindlimb are composed of smaller entities, called neuromuscular compartments, which are the territories of muscle innervated by a single, naturally occurring primary (first-order) muscle nerve branch. While it is quite clear that a precise connectivity exists very early in development between motoneuron pools and individual muscles, the mechanisms responsible for producing the adult pattern of compartmental innervation are unknown. This study uses intracellular recording techniques to demonstrate that neuromuscular compartments are essentially established at birth and that postnatal synapse elimination has little role in establishing neuromuscular compartments. Our results demonstrate the existence of a small number of cross-compartmental connections in neonates which are not present in adults. Examining the removal of these cross-compartmental connections in both normal muscles and in muscles that have had synapse elimination delayed by tenotomy reveals that the synapses responsible for this innervation are eliminated in a selective manner.
\end{abstract}

The rat lateral gastrocnemius muscle is composed of 4 neuromuscular compartments (English and Letbetter, 1982; English et al., 1985; Donahue et al., unpublished observations). Each neuromuscular compartment is defined as the innervation territory of a single, naturally occurring primary muscle nerve branch and has been shown to be composed of an aggregation of single motor units (English and Weeks, 1984; Jannun and English, 1986; Donahue et al., unpublished observations). In the adult, these innervation territories do not overlap. The developmental mechanisms underlying their generation are unknown.

The regressive process referred to as synapse elimination is common in many parts of the developing nervous system. Studies in the visual system (O'Leary and Stanfield, 1985; Stanfield and O'Leary, 1985) and in other regions of the brain (O'Leary et al., 1981; Stanfield et al., 1982; Tolbert and Panneton, 1984; Tolbert, 1987) have demonstrated that the selective loss of certain axon branches during prenatal and early postnatal development refines the topographic projection of the neurons giving rise to these axons. Although it is generally accepted that the projections of motor axons to individual muscles are highly

\footnotetext{
Received May 31, 1988; revised Oct. 12, 1988; accepted Oct. 26, 1988

We are grateful for the critical comments and suggestions of Dr. Donald Wigston and for the technical assistance of Gail Schwartz. This work was supported by USPHS Grants NS 20545 to A.W.E. and RR 00165 to the Yerkes Regional Primate Center.

Correspondence should be addressed to Dr. Arthur English at the above address. Copyright (C) 1989 Society for Neuroscience $0270-6474 / 89 / 051621-07 \$ 02.00 / 0$
}

specific (Landmesser, 1984; Lance-Jones and Landmesser, 1981), some doubt presently exists regarding the role of synapse elimination in refining projections within a given muscle. The results of Brown and Booth (1983a, b) and of Bennett and Lavidis (1984; see also Bennett et al., 1986; Bennett and Ho, 1988) have been used to suggest that a spatial relationship between a motoneuron's position in the spinal cord and its terminal innervation territory in a muscle may be shaped from a less precise innervation pattern by synapse elimination.

In a previous study, we used evoked electromyographic (FMG) activity to demonstrate that the innervation territories of primary muscle nerve branches are generally restricted to their adult pattern long before synapse elimination is complete (Donahue and English, 1987). This finding, as well as those of others (Dennis et al., 1981; English, 1986; Balice-Gordon and Thompson, 1988) suggested that mechanisms occurring prenatally established territories of primary muscle nerve branches in a very specific manner. We did, however, observe a small number of EMG potentials in a denervated neuromuscular compartment during the first postnatal week of life. The study reported on here analyzes the extent and time course of elimination of the synapses responsible for these cross-compartmental potentials. A preliminary report has been published in abstract form (Donahue and English, 1988).

\section{Materials and Methods}

Animals. These experiments used rats from the inbred Fisher 344 strain. Pregnant rats usually gave birth on E22 (P0); the day on which a coital plug was found at 0900 following an overnight mating was designated EO. Postnatal age was determined with the day of birth being designated P0. For experiments evaluating cross-compartmental innervation, 32 animals, evenly distributed from 2 to $12 \mathrm{~d}$ with respect to postnatal and gestational age, were analyzed. Twenty-one animals were unilaterally tenotomized (see below), while 11 served as unoperated controls. Not all muscles were included in the analysis because vigorous muscle contraction did not occur in response to evoked nerve stimulation. The numbers reported in the tables represent those muscles that were used for analysis. For experiments evaluating the extent of polyneuronal innervation, 61 muscles from 42 animals were evaluated. Seventeen animals were tenotomized unilaterally, while 25 served as unoperated controls.

Examination of cross-compartmental innervation. Pups were weighed, heavily ancsthetized with $1.5 \mathrm{mg}$ pentobarbital (i.p.), and perfused through the left ventricle with an oxygenated Ringer's solution of the following composition (mM): $\mathrm{Na}^{+}, 120 ; \mathrm{K}^{+}, 5.0 ; \mathrm{Mg}^{2+}, 1.0 ; \mathrm{Ca}^{2+}, 5.0 ; \mathrm{Cl}^{-}, 117$; $\mathrm{HCO}_{3}^{-}, 20$; glucose, 22; at room temperature. The higher than usual concentration of calcium in the Ringer's solution may help detect additional inputs to skeletal muscle fibers during the period of polyinnervation (Bennett et al., 1986). The perfused animal was pinned prone in a Sylgard-lined dish and immersed in oxygenated Ringer's. The triceps surae muscles (lateral and medial gastrocnemius, and soleus) were removed with their innervation attached as far proximal as the tibial nerve, placed in a Sylgard-lined culture dish, and bathed in oxygenated 


\begin{tabular}{|c|c|c|c|c|c|c|c|c|c|c|c|c|}
\hline \multirow[b]{2}{*}{ Parameter } & \multicolumn{11}{|c|}{ Postnatal age } & \multirow[b]{2}{*}{ Total } \\
\hline & 2 & 3 & 4 & 5 & 6 & 7 & 8 & 9 & 10 & 11 & 12 & \\
\hline \multicolumn{13}{|l|}{ Number of muscles examined } \\
\hline Tenotomy & 3 & 3 & 2 & 3 & 3 & 1 & 1 & 2 & 2 & 0 & 0 & 20 \\
\hline Normal & 3 & 3 & 2 & 3 & 2 & 3 & 2 & 2 & 5 & 2 & 2 & 29 \\
\hline Total & 6 & 6 & 4 & 6 & 5 & 4 & 3 & 4 & 7 & 2 & 2 & 49 \\
\hline Number of impaled cells in & & & & & & & & & & & & \\
\hline $\mathrm{LGm}^{a}$ & 108 & 120 & 80 & 114 & 88 & 89 & 60 & 92 & 132 & 40 & 47 & 970 \\
\hline $\begin{array}{l}\text { Percent of cells with cross- } \\
\text { compartmental innervation }\end{array}$ & 8 & 13 & 4 & 11 & 8 & 3 & 0 & 1 & 0 & 0 & 0 & 5.3 \\
\hline
\end{tabular}

Ringer's solution. The nerve to lateral gastrocnemius and soleus (LGS) was dissected to expose the primary branch to the medial head of LG (LGm) and this branch was cut. In the adult, muscle fibers in LGm are innervated exclusively by axons coursing in this primary branch, and hence LGm is one of the 4 distinct neuromuscular compartments of LG. All muscle fibers innervated exclusively by axons coursing in the primary branch to LGm were denervated by cutting this branch. The remainder of $\mathrm{LG}$ was then activated by supramaximal stimulation $(0.1-0.5 \mathrm{~mA} ; 0.05 \mathrm{msec})$ of the LG-S nerve. Muscles were not paralyzed in order to increase the likelihood of detecting inputs, and thus all fibers not innervated exclusively by the branch to $\mathrm{LGm}$ were presumably activated. The LG muscle was transilluminated to visualize its tendons and fascicle architecture, as well as to verify the location of the recording electrode on the muscle. Since the 3 tendons of LG also serve as boundaries for the innervation territories of the primary muscle nerve branches, by viewing the transilluminated muscle we could be certain of the neuromuscular compartment in which the impaled cell was located. Intracellular impalements were made with glass microclectrodes (rcsistances of 40-80 M $\Omega$ when filled with $3 \mathrm{M} \mathrm{KCl}$ ) into regions of muscle near the endplate zone, although endplates were not visualized in the preparation. The muscle was stretched and pinned to the dish to reduce muscle contraction; each head of LG was stretched to approximately the same degree. We found that this stretching caused depolarization of muscle cells, so we accepted any penetration with a stable resting membrane potential equal to or more negative than $-40 \mathrm{mV}$. For each impaled cell, we observed the response to LG-S nerve stimulation. The muscle was not paralyzed, and the resulting contraction usually dislodged the electrode from the muscle cell. The latency to onset of any action potentials recorded was noted. The amplitude and rise time of any observed endplate potentials (EPPs) were also recorded. In all muscles, at least 24 cells were impaled. Twenty cells were located in the LGm head, while the remaining 4 were distributed throughout the other 3 compartments in the remaining 2 heads of LGm (Donahue and English, 1987). We were prepared to exclude from analysis all cases in which potentials could not be observed in the innervated lateral or intermediate heads; this occurred on only 5 occasions; over $90 \%$ of the time the first cell impaled in an innervated head showed evidence for an input. We calculated the percentage of cross-compartmental potentials and expressed it as a function of gestational age, postnatal age, and body weight.

Examination of polyneuronal innervation. The extent of polyneuronal innervation in rat LG was examined using graded nerve stimulation (see Redfern, 1970). Forty-four normal muscles and 17 tenotomized muscles from animals aged P2-P13 were removed as described above and paralyzed using either $10^{-6} \mathrm{M} d$-tubocurarine or $2 \mathrm{M}$ formamide (Donahue and English, 1989). Formamide paralysis was used strictly for animals younger than P8, while $d$-tubocurarine was used in all older animals. Intracellular penetrations using 40-80 M electrodes were performed, and graded nerve stimulation was used to evaluate the number of inputs to penetrated muscle fibers. Each stepwise increase in EPP amplitude that had a consistent amplitude and stimulus threshold was judged to result from the activation of the fiber by a single axon (Redfern, 1970).

Tenotomy. Tenotomy has been shown to delay synapse elimination in both rat and cat soleus; both the average number of inputs (Riley, 1978), and the percentage of polyinnervated cells (Benoit and Changeux,
1975) are greater in tenotomized muscles than in contralateral control muscles. To observe the effect of tenotomy on the elimination of crosscompartmental innervation, newborn (P0) rats were deeply anesthetized with methoxyflurane, and a dorsal incision was made in the skin over the surface of one calcaneus to expose the tendocalcaneus. A small $(\sim 500 \mu \mathrm{m})$ piece of the tendocalcaneus was then removed, being careful not to damage the muscle fibers. The skin over the wound was opposed with a 7-0 silk suture, and the animal was placed on a heating pad until it recovered sufficiently (spontaneous movements) to be returned to its mother. The animal was ususally isolated from its mother for less than $15 \mathrm{~min}$. The contralateral muscle served as an unoperated control. After various survival times, animals were anesthetized and studied as described above. Both the tentomized and control muscles were analyzed to determine the extent of cross-compartmental innervation and the extent of polyneuronal innervation. Since the tendocalcaneus rapidly reattaches, most often to its normal insertion, the procedure was repeated on postoperative days 3 and 7 in animals still living at those timcs.

\section{Results}

\section{Innervation during polyinnervation is generally compartment-specific}

We examined the terminal distribution of axons coursing in individual primary muscle nerve branches by cutting the primary branch serving one neuromuscular compartment and recording from muscle cells in that compartment in response to supramaximal stimulation of the remaining primary branches. There was no difference in the amount of cross-compartmental innervation seen between muscles of normal animals and the contralateral control muscles of tenotomized animals. In nearly $95 \%$ of 970 cells in the LGm compartment of 49 muscles from animals aged 2-12 d, no potentials could be evoked when stimulating the remaining primary muscle nerve branches after cutting the branch to the LGm compartment (Table 1). In nondenervated compartments, over $95 \%$ of cells demonstrated evoked potentials upon supramaximal stimulation. The remaining small percentage of cells that did not have potentials were likely denervated or damaged either during the dissection or during the intracellular impalement. No factor was introduced into our calculations to account for this observation. During the first postnatal week, $8.3 \%$ of sampled cells received cross-compartmental innervation. No cross-compartmental innervation was seen in normal muscles after postnatal day seven. When muscles from animals of all postnatal ages were taken into account, only $5.3 \%$ of impaled cells showed evidence for cross-compartmental innervation (Table 1). Thus, the main conclusion from this study is that muscles are innervated in a compartment-specific manner at birth and that postnatal synapse elimination generally does not serve to establish neuro- 


\section{TENOTOMY AND SYNAPSE ELIMINATION}
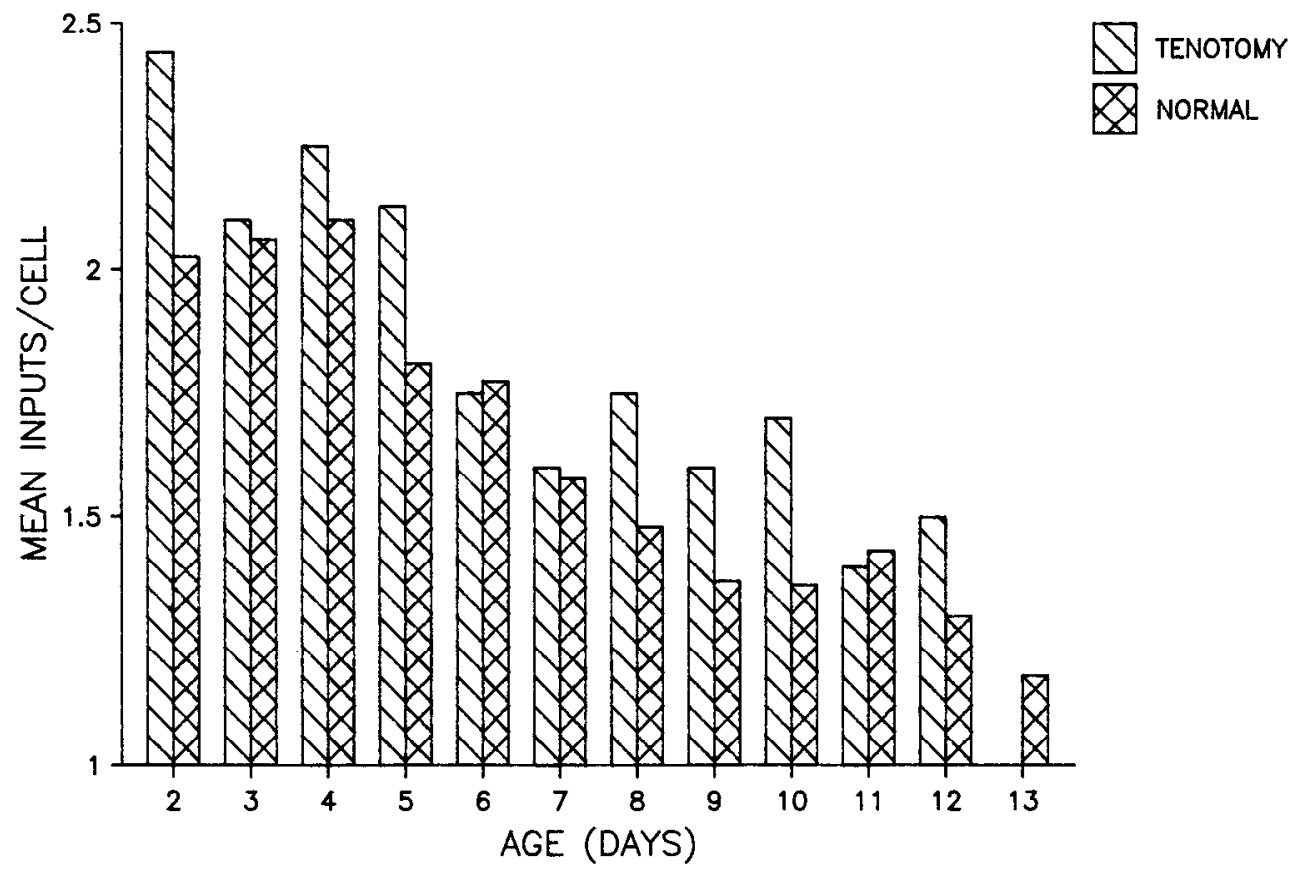

muscular compartments. This is in support of our previous studies using evoked electromyography (Donahue and English, 1987) and suggests that neuromuscular compartments are essentially established by a process occurring before the time of birth.

\section{A small amount of cross-compartmental innervation does exist}

The results presented above clearly demonstrate that a small number of muscle fibers in LGm receive inputs from axons that course in a primary nerve branch other than the primary branch to LGm. Almost all (50 of 51) cells with cross-compartmental innervation were from animals $0-7 \mathrm{~d}$ of age. In some muscles, however, cross-compartmental innervation was much more prevalent. Although we studied 31 muscles during the first postnatal week, in only 23 of them did we sample cells with crosscompartmental innervation. In these muscles, $11.9 \%$ of $\mathrm{LGm}$ cells received cross-compartmental innervation. There was no difference in the extent of cross-compartmental innervation between tenotomized muscles and contralateral control muscles.

Our experiments were complicated because muscle contraction prevented us from repeatedly observing cross-compartmental potentials in the same muscle cell. However, we felt that maximizing the probability of detecting weak inputs was of sufficient importance that analyzing the extent of cross-compartmental innervation in muscles paralyzed with curare or formamide was not attempted. The resting membrane potentials of cells in LGm did not differ from the potentials of cells in neighboring compartments. The rise times and latencies to onset of the cross-compartmental evoked potentials were also similar to those of EPPs seen in innervated compartments, suggesting that these potentials did not result from intercellular coupling between myotubes (see Discussion).

\section{Tenotomy delays compartmental synapse elimination}

On postnatal day $(\mathrm{P} 2)$, skeletal muscle fibers in rat $L G$ receive an average of 2.1 inputs (Fig. 1). The number of inputs/cell decreases in a relatively linear manner from $\mathrm{P} 4$ through P13. Muscle fibers from tenotomized muscles receive greater amounts of polyinnervation than do fibers from contralateral control muscles. While the difference in the extent of polyneuronal innervation between tenotomized and normal muscles at each postnatal age is not significant (because of the small sample size), a difference can be demonstrated by using a paired $t$ test. In the 15 unilaterally tenotomized animals, the mean difference in the average number of inputs per cell observed between tenotomized and contralateral control muscles $(0.26 \pm 0.07$; mean \pm SEM; $n=15)$ was statistically significant ( $p<0.005$; paired $t$ test).

\section{Time course of elimination of cross-compartmental innervation}

The percentage of cross-compartmental innervation detected at various times during synapse elimination for both tenotomized and normal muscles is expressed in Figure 2. Neither the slopes nor the intercepts of the least-squares linear-regression lines were significantly different between tenotomized and normal muscles. However, the variance of the points used to determine these lines was quite large, as evidenced by the small correlation coefficients $(r=-0.563$ for normal muscles; $r=-0.423$ for tenotomized muscles). Analyzing these data with log transformations did not reduce this variance (data not shown). With the exception of one cell in a tenotomized muscle that received cross-compartmental innervation at P9 (see Discussion), no cross-compartmental potentials were detected in any LGm cells from animals aged $7 \mathrm{~d}$ or older.

While tenotomy delays the elimination of polyinnervation both in soleus (Benoit and Changeux, 1975; Riley, 1978) and in LG (Donahue et al., unpublished observations), it does not delay significanlty the elimination of cross-compartmental innervation. For the 7 muscle pairs from unilaterally tenotomized animals where some cross-compartmental innervation was detected in at least one muscle, the mean increase in cross-com- 
Figure 2. Extent of cross-compartmental innervation varies as a function of postnatal age in both tenotomized $(\times)$ and normal (squares) muscles.

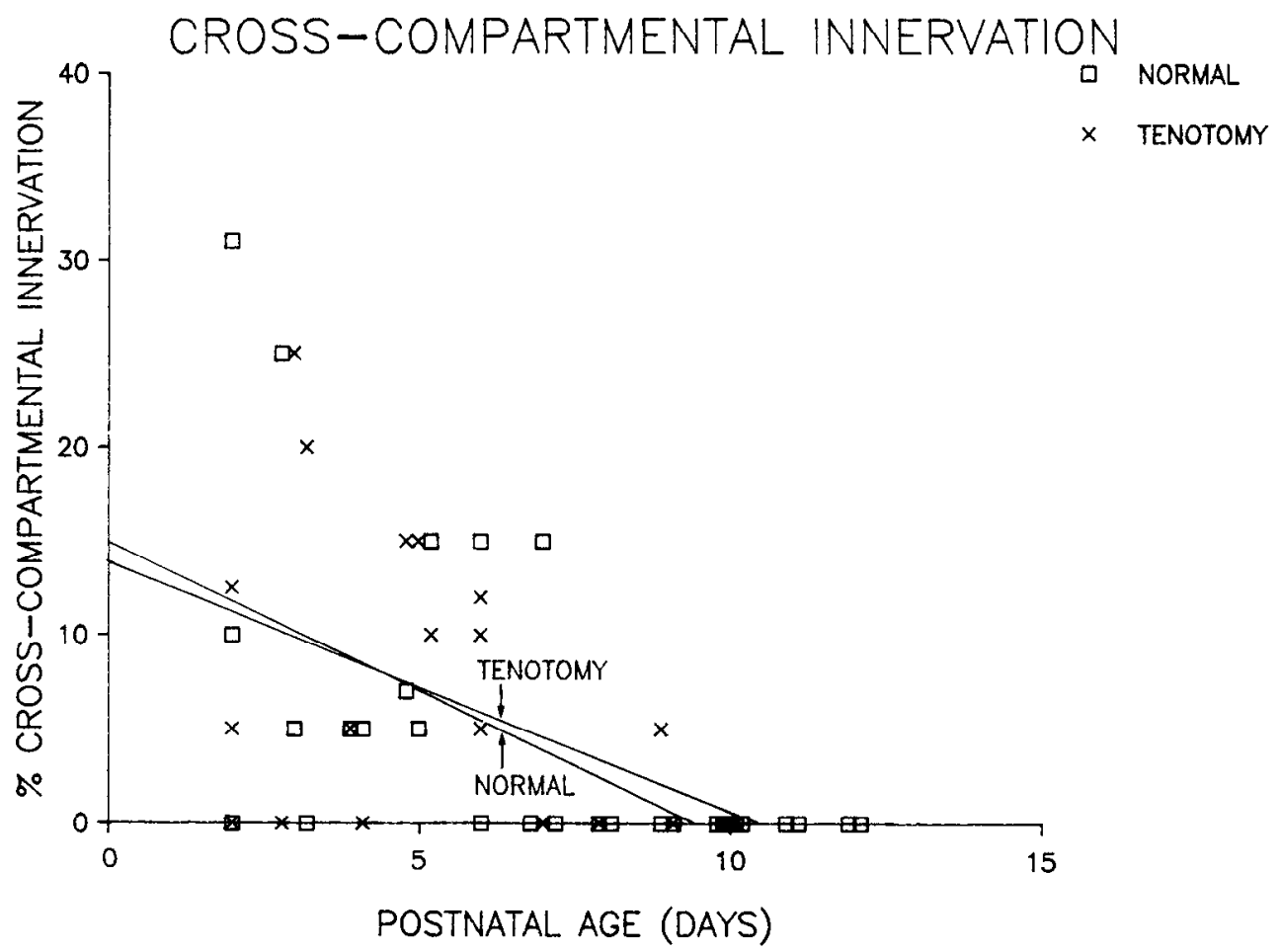

partmental innervation observed in the tenotomized muscles $(3$ $\pm 4 \%$ ) was not significantly different from zero $(p=$ NS; paired $t$ test).

Figure 3 compares the elimination of cross-compartmental innervation with that of compartmental innervation. For each day from $\mathrm{P} 3$ to $\mathrm{P} 13$, the amount of elimination that has occurred, relative to that observed on $P 2$, is given. With one exception (P5), more cross-compartmental innervation than compartmental innervation has been eliminated on each postnatal day.

\section{Cross-compartmental innervation is confined to certain regions of $L G m$}

To test whether cross-compartmental innervation was distributed evenly within LGm, we subdivided the LGm head into proximal, central, and distal areas, and compared the relative amounts of cross-compartmental innervation observed in each location using a $\chi^{2}$ test. In a $\chi^{2}$ analysis, the actual occurrence of an event is compared with its expected likelihood of occurrence given a particular assumption, in this case, that crosscompartmental innervation is distributed uniformly within LGm. If the expected and observed frequencies differ significantly, then the assumption is rejected. The results of this partitioning of LGm are displayed in Table 2 , and the $\chi^{2}$ analysis is summarized in Table 3 . Since there was no difference in the results between tenotomized and normal muscles, the data were pooled. The expected amount of cross-compartmental innervation in each region is the overall percentage of cross-compartmental innervation seen in LG muscles having cross-compartmental innervation $(12.6 \%)$. While only $4.8 \%$ of 104 cells sampled in the proximal portion of $\mathrm{LGm}$ received cross-compartmental innervation, $14.6 \%$ of 157 cells impaled in the central region of LGm and $17.9 \%$ of the 95 cells sampled in the distal part of LGm received inputs from axons that coursed in a branch other than the primary branch to LGm. The $F$-statistic (8.7) was significant $(p<0.02)$, rejecting the hypothesis that the innervation is evenly distributed throughout the compartment.

\section{Discussion}

The results presented here confirm that the innervation territories of compartmental nerve branches are largely established at birth and that postnatal synapse elimination is not shaping compartmental innervation patterns from a more diffuse pattern. We do show, however, that approximately $5 \%$ of synapses onto fibers in a neuromuscular compartment originate from axons that course in a primary nerve branch to an adjacent compartment and then cross a compartment boundary. Three arguments can be marshalled against the hypothesis that the elimination of cross-compartmental axon branches occurs by the same process that eliminates compartmental synapses. Instead, these arguments suggest that selective mechanisms are involved in the loss of cross-compartmental innervation.

Table 2. Location of cross-compartmental innervation ${ }^{a}$

\begin{tabular}{lcllllll}
\multirow{2}{*}{$\begin{array}{l}\text { Region } \\
\text { LGm }\end{array}$} & \multicolumn{9}{l}{ Postnatal age } & & \\
\cline { 2 - 6 } Troximal & 2 & 3 & 4 & 5 & 6 & 7 & Total \\
\hline Pentral & $1 / 22$ & $2 / 18$ & $0 / 13$ & $0 / 23$ & $2 / 24$ & $0 / 4$ & $5 / 104$ \\
& $3 / 27$ & $8 / 44$ & $2 / 25$ & $5 / 23$ & $4 / 29$ & $1 / 9$ & $23 / 157$ \\
Distal & $(11)$ & $(18)$ & $(8)$ & $(22)$ & $(14)$ & $(11)$ & $(14.69)$ \\
& $6 / 20$ & $4 / 17$ & $0 / 21$ & $4 / 14$ & $1 / 16$ & $2 / 7$ & $17 / 95$ \\
& $(30)$ & $(24)$ & $(0)$ & $(29)$ & $(6)$ & $(29)$ & $(17.9)$
\end{tabular}

a No observed difference between tenotomy and normal; results pooled. The regional location of the cell with the cross-compartmental innervation was recorded in 45 of the 51 cells having cross-compartmental innervation. This table reflects only those experiments where the intracompartmental location of the impaled cells was recorded. The percentage of muscles having cross-compartmental innervation is shown beneath the ratio (number of cells with cross-compartmental innervation/ total number of impaled cells). 


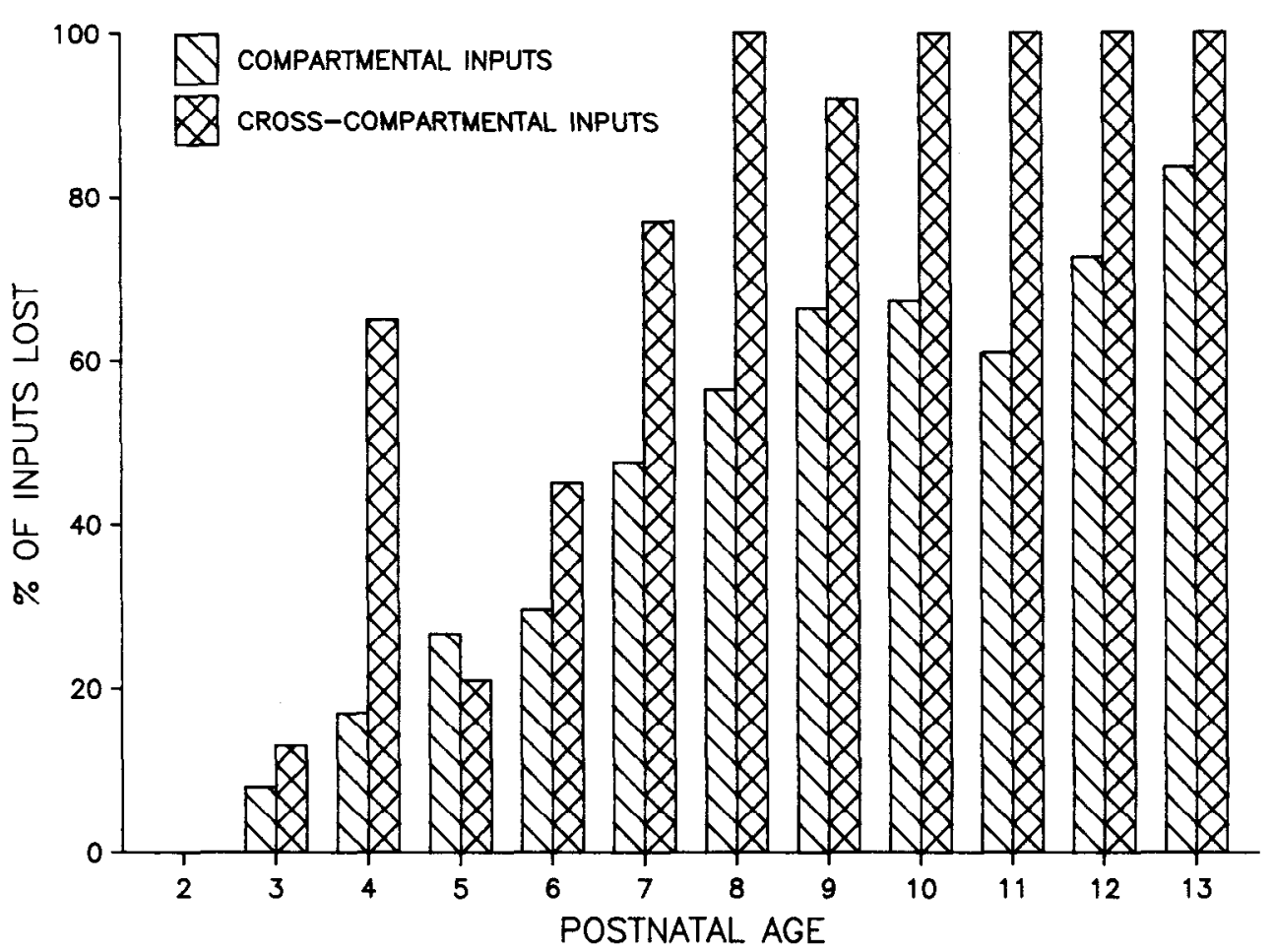

Figure 3. Cross-compartmental innervation is eliminated earlier than compartmental innervation. For compartmental inputs (hatched bars), each bar represents the percentage of inputs lost at each day relative to the total number of inputs that need to be eliminated on P2 to produce $100 \%$ monoinnervation (data from Donahue et al., 1988). For cross-compartmental inputs (cross-hatched bars), each bar represents the percentage difference between the proportion of cross-compartmental inputs on a given day and the proportion on $\mathrm{P} 2$. While nearly all cross-compartmental innervation is eliminated before postnatal day 8 , compartmental synapse elimination continues into the third postnatal week of life.
First, we could not observe any cross-compartmental innervation in muscles from normal animals older than 7 postnatal days. If cross-compartmental innervation were lost at a rate similar to that of compartmental innervation, small amounts of cross-compartmental innervation should be detected throughout the time period during which synapse elimination occurs. We impaled over $230 \mathrm{LGm}$ cells in 13 normal muscles from animals aged 8-12 d and never observed a cross-compartmental endplate potential or action potential. Even if the true extent of cross-compartmental innervation during this time period is only $2 \%$ (considerably less than the average crosscompartmental innervation observed up until this time), the probability of impaling 230 cells and never observing a potential is less than 0.005 .

Second, the rate of loss of normal synapses is different from the rate of loss of cross-compartmental innervation. When expressed as a percentage of the synapses that are to be eliminated, a relativcly grcatcr proportion of cross-compartmental synapses have been lost on each postnatal day compared with compart- mental synapses (Fig. 3). Finally, tenotomy delays compartmental synapse elimination (Benoit and Changeux, 1975; Riley, 1978; Fig. 1) but does not appear to delay the loss of crosscompartmental innervation (Fig. 2). While we cannot explain why one tenotomized muscle from a 9-d-old animal showed cross-compartmental innervation in one cell, it is possible that the tenotomy procedure stimulated axonal sprouting into LGm by axons in adjacent compartments by damaging or denervating some of the fibers. Although our results demonstrate that crosscompartmental synapses are lost selectively, presumably because of their aberrant location in the muscle, this selective elimination is not the same as the proposed selective loss of axons based on their segmental origin, as suggested by Bennett and Lavidis (1984).

It is possible that the observed cross-compartmental potentials might result from the spread of electrical current through gap junctions which might connect muscle fibers on opposite sides of compartment boundaries during the first postnatal week, but this is unlikely. The cross-compartmental potentials were

Table 3. Location of cross-compartmental innervation in LGm

\begin{tabular}{|c|c|c|c|c|c|}
\hline \multirow[b]{2}{*}{ Region } & \multicolumn{2}{|c|}{$\begin{array}{l}\text { Cells having cross- } \\
\text { compartmental innervation }\end{array}$} & \multicolumn{2}{|c|}{$\begin{array}{l}\text { Cells without cross- } \\
\text { compartmental innervation }\end{array}$} & \multirow[b]{2}{*}{ Total } \\
\hline & Observed & $\left(\right.$ Expected $\left.^{a}\right)$ & Observed & (Expected $\left.^{a}\right)$ & \\
\hline Proximal & 5 & (13) & 99 & (91) & 104 \\
\hline Central & 23 & (20) & 134 & (137) & 157 \\
\hline Distal & 17 & (12) & 78 & (83) & 95 \\
\hline Totals & 45 & & 31 & & 356 \\
\hline
\end{tabular}

$F$ statistic $\quad 8.7, p<0.02, v=2,44$

${ }^{a}$ Calculated as follows: We observed 45 instances of cross-compartmental innervation during 356 impalements (12.6\%) of muscles having some cross-compartmental innervation where a location in LGm was recorded (see Table 3 ). Thus, for each region, the expected frequency of cross-compartmental innervation was $12.6 \%$ of the number of cells impaled in that region. The expected value is given in parentheses to the right of the observed value. 
not restricted to specific areas near the border of the LGm com partment, which is where they might be if they resulted from intracellular coupling to cells in adjacent compartments. Dennis et al. (1981) have suggested that endplate potentials caused by gap junctional coupling between adjoining segments have a slow rise time, but we did not observe any difference in latency or rise time of cross-compartmental potentials relative to compartmental potentials. Furthermore, the observations of Dennis et al. could only be made in fetal muscles. Electron microscopic evidence for gap junctional coupling has been observed during the first postnatal week (see Schmalbruch, 1982) but involves a primary myotube and the newly differentiating, uninnervated secondary myotubes that form along its walls (Ontell and Dunn, 1978). Since primary and secondary myotubes are found in the same muscle fascicle in adults, and since all cells in a fascicle are in the same neuromuscular compartment (Donahue and English, unpublished observations), this type of coupling also could not produce cross-compartmental innervation. The spread of current, either from the stimulating electrode or from activated muscle cells, might also produce artifactual coupling. However, similar arguments as those presented above also make this possibility unlikely: potentials were not observed in consistent locations such as near tendinous boundaries or the stimulating electrode. Furthermore, the duration of the current pulses used was only $50 \mu \mathrm{sec}$, and our experiments have indicated that stimulus durations considerably longer than this are needed to excite cells by means of volume conduction.

The observed cross-compartmental potentials thus result from axons that course in a primary nerve branch other than the one to LGm, and cross the compartment boundary to innervate fibers in the LGm compartment. If some axons make synapses in a relatively diffuse manner throughout what will be their adult innervation territory, some axons could enter the medial head. The proximal portion of LGm is separated from the adjacent LG compartment by a deep tendon of origin of $L G$, which ends midway distally in LG (Donahue and English, 1987; Donahue et al., unpublished observations). After the tendon of origin ends, however, no physical boundary exists between the distal portions of LGm and LGi. In the absence of this border, axons ramifying within the distal LGi might extend terminals into the adjacent distal LGm. This might explain the overabundance of cross-compartmental innervation within the distal portion of LGm (Table 3). This regional distribution of cross-compartmental innervation also stresses the importance of sampling cells at various locations throughout a muscle during intracellular experiments.

It is possible that the extent of cross-compartmental innervation in very young animals is considerably larger than we observed, as our results suggest that a relatively large number of cells in the distal part of LGm might receive cross-compartmental innervation during early stages of polyinnervation (Table 2). Our results might underestimate cross-compartmental innervation in very young animals if cutting a single primary muscle nerve branch damages some axons that provide this innervation. However, if axons are damaged by the dissection, our conclusion favoring selective loss of cross-compartmental innervation is strengthened: First, the larger muscles of P8-P12 animals are relatively easy to denervate partially but had no cross-compartmental innervation. Second, the cross-compartmental innervation would need to be lost even more rapidly during early postnatal synapse elimination to produce the distinct compartment boundaries we observed by $\mathbf{P} 7$.
We are unsure why cross-compartmental innervation is lost selectively. Several experiments have demonstrated that selective reinnervation of muscles can occur (Wigston and Sanes, 1982, 1985; Laskowski and Sanes, 1987; Wigston and Kennedy, 1987). It has been suggested that muscles possess intrinsic recognition cues that promote selective reinnervation, even when muscles are transplanted to novel locations (Wigston, 1986) and that some of this selectivity can be abolished by destroying a muscle's fibers (Wigston and Sanes, 1985; Wigston and Donahue, 1988). It is possible that these postulated recognition cues are generally compartment-specific and that this specificity underlies the selective innervation of neuromuscular compartments that we have observed at birth. We are unsure, however, why inherently unstable synapses would be maintained well past the time of birth. Nevertheless, a minor degree of mismatch between pre- and postsynaptic elements could account for both the small proportion of cross-compartmental innervation observed by us. The relative instability of mismatched synapses might underlie their selective elimination.

\section{References}

Balice-Gordon, R. J., and W. J. Thompson (1988) The organization and development of compartmentalized innervation in rat extensor digitorum longus muscle. J. Physiol. (Lond.) 395: 211-232.

Bennett, M. R., and S. Ho (1988) The formation of topographical maps in developing rat gastrocnemius muscle during synapse elimination. J. Physiol. (Lond.) 396: 471-496.

Bennett, M. R., and N. A. Lavidis (1984) Development of the topographical projection of motor neurons to a rat muscle accompanies loss of polyneuronal innervation. J. Neurosci. 4: 2204-2212.

Bennett, M. R., S. Ho, and N. Lavidis (1986) Competition between segmental nerves at endplates in rat gastrocnemius during loss of polyneuronal innervation. J. Physiol. (Lond.) 381: 351-376.

Benoit, P., and J. P. Changeux (1975) Consequences of tenotomy on the evolution of multiinnervation in developing rat soleus muscle. Brain Res. 99: 354-358.

Brown, M. C., and C. M. Booth (1983a) Postnatal development of the adult pattern of motor axon distribution in rat muscle. Nature 304: 741-742.

Brown, M. C., and C. M. Booth (1983b) Segregation of motor nerves on a segmental basis during synapse elimination in neonatal muscles. Brain Res. 273: 188-190.

Dennis, M. J., L. Ziskind-Conhaim, and A. J. Harris (1981) Development of neuromuscular junctions in rat embryos. Dev. Biol. 81 : 266-279.

Donahue, S. P., and A. W. English (1987) The role of synapse elimination in the establishment of neuromuscular compartments. Dev. Biol. 124: 481-489.

Donahue, S. P., and A. W. English (1988) Selective elimination of cross-compartmental innervation during synapse elimination. Soc. Neurosci. Abstr. 14: 179.

Donahue, S. P., and A. W. English (1989) Formamide increases the number of detectable inputs to developing neuromuscular junctions. J. Neurosci. Methods (in press).

Donahue, S. P., J. G. Wood, and A. W. English (1987) Delayed expression of $200 \mathrm{~K}$ NFP at the neuromuscular junction. Soc. Neurosci. Abstr. 13: 1425.

Donahue, S. P., R. L. Roden, G. A. Schwartz, and A. W. English (1988) Tenotomy delays secondary myogenesis. Anat. Rec. 220:33A.

English, A. W. (1986) Does synapse elimination shape neuromuscular compartments? Soc. Neurosci. Abstr. 12: 1118.

English, A. W., and W. D. Letbetter (1982) Anatomy and innervation patterns of cat lateral gastrocnemius muscle. Am. J. Anat. 164: 6777.

English, A. W., and O. I. Weeks (1984) Compartmentalization of single muscle units in cat lateral gastrocnemius. Exp. Brain Res. 56: 361368.

English, A. W., S. P. Donahue, and O. I. Weeks (1985) Compartmentalization of rat lateral gastrocnemius muscle. Am. Zool. 25: 107A. 
Jannun, D., and A. W. English (1986) Compartmentalization of single muscle units in the rat lateral gastrocnemius. Anat. Rec. 214:60A.

Kugelberg, E., L. Edstrom, and M. Abbruzzese (1970) Mapping of motor units in experimentally reinnervated muscle. J. Neurol. Neurosurg. Psychol. 33: 319-329.

Lance-Jones, C., and L. T. Landmesser (1981) Pathway selection by chick lumbosacral motoneurons during normal development. Proc. R. Soc. Lond. [Biol.] 214: 1-18.

Landmesser, L. (1984) The development of specific motor pathways in the chick embryo. Trends Neurosci. 7: 336-339.

I askowski, M. B., and J. R. Sanes (1987) Topographic mapping of motor pools onto skeletal muscles. J. Neurosci. 7: 252-260.

O'Leary, D. D. M., and B. B. Stanfield (1985) Occipital cortical neurons with transient pyramidal tract axons extend and maintain collaterals to subcortical but not intracortical targets. Brain Res. 336: 326-333.

O'Leary, D. D. M., B. B. Stanfield, and W. M. Cowan (1981) Evidence that the early postnatal restriction of the cells of origin of the collosal projection is due to the elimination of axonal collaterals rather than to the death of neurons. Dev. Brain Res. 1: 607-617.

Ontell, M. A., and R. F. Dunn (1978) Neonatal muscle growth: A quantitative study. Am. J. Anat. 152: 539-556.

Redfern, P. A. (1970) Neuromuscular transmission in newborn rats. J. Physiol. (Lond.) 209: 701-709.

Riley, D. A. (1978) Tenotomy delays the postnatal development of the motor innervation of the rat soleus. Brain Res. 143: 162-167.

Schmalbruch, H. (1982) Skeletal muscle fibers of newborn rats are coupled by gap junctions. Dev. Biol. 91: 485-490.
Stanfield, B. B., and D. D. M. O'Leary (1985) The transient corticospinal projection from the occipital cortex during the postnatal development of the rat. J. Comp. Neurol. 238: 236-248.

Stanfield, B. B., D. D. M. O'Leary, and C. Fricks (1982) Selective collateral elimination in early postnatal development restricts cortical distribution of rat pyramidal tract neurones. Nature 298: 371-373.

Tolbert, D. L. (1987) Intrinsically directed pruning as a mechanism regulating the elimination of transient collateral pathways. Dev. Brain Res. 33: 11-21.

Tolbert, D. L., and W. M. Panneton (1984) The transience of cerebrocerebellar projections is due to selective elimination of axon collaterals and not neuronal death. Dev. Brain Res. 16: 301-306.

Wigston, D. J. (1986) Selective innervation of transplanted limb muscles by regenerating motor axons in the axolotl. J. Neurosci. 6: $2757-$ 2763.

Wigston, D. J., and S. P. Donahue (1988) The location of cues promoting selective reinnervation of axolotl muscles. J. Neurosci. 8 : $3451-3458$.

Wigston, D. J., and P. R. Kennedy (1987) Selective reinnervation of transplanted muscles by their original motoneurons in the axolotl. J. Neurosci. 7: 1857-1865.

Wigston, D. J., and J. R. Sanes (1982) Selective reinnervation of adult mammalian muscle by axons from different segmental levels. Nature 299: 464-467.

Wigston, D. J., and J. R. Sanes (1985) Selective reinnervation of intercostal muscles transplanted from different segmental levels to a common site. J. Neurosci. 5: 1208-1221. 